\title{
The ultrasound-guided retrolaminar block: volume-dependent injectate distribution
}

This article was published in the following Dove Press journal: Journal of Pain Research

\author{
Marija Damjanovska' \\ Tatjana Stopar Pintaric ${ }^{1,2}$ \\ Erika Cvetko \\ Kamen Vlassakov ${ }^{3}$ \\ 'Clinical Department of \\ Anesthesiology and Intensive \\ Therapy, University Medical Centre \\ Ljubljana, Ljubljana, Slovenia; \\ 2Institute of Anatomy, Faculty of \\ Medicine, University of Ljubljana, \\ Ljubljana, Slovenia; ${ }^{3}$ Department of \\ Anesthesiology, Perioperative and \\ Pain Medicine, Brigham and Women's \\ Hospital, Harvard Medical School, \\ Boston, MA, USA
}

Purpose: The ultrasound-guided retrolaminar block is one of the newer and simpler alternatives to the traditional, often technically challenging, paravertebral (PV) block. Its feasibility, safety, and efficacy have already been clinically demonstrated in patients with multiple rib fractures using higher volumes of local anesthetic, when compared with the traditional approach. The primary aim of this observational anatomical study was to assess the spread of local anesthetic from the retrolaminar injection point to the PV space and its volume dependence. Second, we assessed the incidence of epidural and contralateral PV spread in the both groups.

Methods: Ten fresh porcine cadavers were randomized into 2 groups ( $\mathrm{n}=5$ each) to receive ultrasound-guided retrolaminar injections at Th4-Th5 level with either $10 \mathrm{~mL}$ (low-volume group) or $30 \mathrm{~mL}$ (high-volume group) of $2 \%$ lidocaine and methylene blue mixture. After the procedure, the cadavers were dissected and frozen. Cross-section cuts $(\sim 1 \mathrm{~cm}$ thick) were performed to evaluate the injectate spread.

Results: In the high-volume group, injectate spread from the retrolaminar to the PV space was observed in all specimens ( 5 out of $5 ; 100 \%$ ), while in the low-volume group, no apparent spread to the PV space was found ( 0 out of $5 ; 0 \%$ ). No epidural or contralateral PV spread was observed in any of the specimens.

Conclusion: Following ultrasound-guided retrolaminar injections in fresh porcine cadavers, injectate spread from the retrolaminar tissue plane to the PV space is strongly volume dependent, suggesting that, clinically, high local anesthetic volumes maybe critical for achieving regional anesthesia and analgesia consistent with traditional PV blockade.

Keywords: paravertebral space, vertebral lamina, local anesthetic, injections

\section{Introduction}

Optimal pain management is a priority in patients with multiple rib fractures as it prevents pulmonary complications associated with significant morbidity and mortality, especially in the elderly population. ${ }^{1}$ Thus far, both thoracic epidural and paravertebral (PV) analgesia have been used, demonstrating comparably effective pain relief. . $^{2,3}$ However, the PV blockade (PVB) is associated with greater hemodynamic stability, ${ }^{4}$ particularly important in potentially unstable trauma patients where a mild to moderate degree of impaired hemostasis may not amount to a regional block contraindication. ${ }^{3}$ Nevertheless, anticipated major bleeding and subsequent major coagulation disorders still limit the wide PVB utility in these settings. Though very small, the risks of pneumothorax and inadvertent epidural and intrathecal drug administration also remain serious considerations. . $^{5,6}$
Correspondence: Marija Damjanovska Clinical Department of Anesthesiology and Intensive Therapy, University Medical Centre Ljubljana, Zaloska 7, 1000 Ljubljana, Slovenia

Tel +386 4l 990745

Email marijadamjanovska@gmail.com 
Ultrasound guidance offers a potential benefit of reducing these complications and increasing the success rate because of the real-time visualization of the PV space, surrounding structures, the tip of the approaching needle, and the spread of local anesthetic. ${ }^{7}$ So far, however, there has been no study conclusively supporting such claims. ${ }^{8}$ The sonographic window is typically rather limited, often precluding optimal PV space image acquisition while the block needle is advanced in the immediate proximity to both pleura and intervertebral foramina. Therefore, the risk of pleural puncture and even the possibility of epidural or intrathecal spread of local anesthetics are still present. ${ }^{7,9}$

Ultrasound-guided retrolaminar block is one of the newer and technically simpler alternatives to the traditional PV block. The first case report by Pfeiffer et $\mathrm{al}^{10}$ of a landmark lamina technique was followed by a study in mastectomy patients ${ }^{11}$ where this "blind" technique was used to inject local anesthetic between the thoracic laminae and the deep paraspinous muscles. This technique would logically decrease the chances of pleural injury, while the risk of inadvertent neuraxial injection remains. In 2013, Zeballos et a ${ }^{12}$ suggested improving the lamina technique by mandating ultrasound guidance, defining the site of injection as "retrolaminar" by real-time sonographic identification of the lamina and the needle tip-lamina contact, thus minimizing the risk of epidural injection associated with the blind technique. This was immediately followed by a report by the same group describing their first experiences with the retrolaminar technique in patients with multiple rib fractures. ${ }^{13}$

The feasibility, efficacy, and safety of the ultrasoundguided retrolaminar block have been clinically demonstrated in trauma patients, notably using higher local anesthetic volumes than those needed with the traditional approach. ${ }^{12-16}$ However, there is still very limited knowledge about the actual distribution of retrolaminar "plane" injectates and its dependence on volume. In fact, only one study ${ }^{16}$ mentions a pilot experiment determining that " $20 \mathrm{~mL}$ was more satisfactory than 10 or 15 $\mathrm{mL}$ for retrolaminar block and that $25 \mathrm{~mL}$ was equivalent to $20 \mathrm{~mL}$ " in patients undergoing radical mastectomy.

The primary aim of this study was to evaluate the spread of local anesthetic/dye mixture from the retrolaminar injection point to the $\mathrm{PV}$ space and assess its volume dependence in a porcine model. A secondary aim was to assess the incidence of epidural and contralateral PV spread of local anesthetic/dye mixture in studied groups.

\section{Methods}

Ten fresh porcine cadavers (from animals that had been previously used for another study ${ }^{17}$ and then euthanized) were used as approved by Review Board for Animal Research (No. U34401-28/2013/17). The previous protocol had not affected the cadavers used in this study in any substantial way.

The porcine cadavers weighing around $20 \mathrm{~kg}$ were randomly allocated into 2 groups ( $\mathrm{n}=5$ each) to receive unilateral ultrasound-guided retrolaminar injections at Th4-Th5 level with either $10 \mathrm{~mL}$ (low-volume group) or $30 \mathrm{~mL}$ (highvolume group) of $2 \%$ lidocaine (Lekarna UKC Ljubljana, Ljubljana, Slovenia) and methylene blue mixture (Lekarna UKC Ljubljana).

The animals were placed in left recumbent position. The retrolaminar space was identified sonographically (9L-RS, 3.1-10.0 MHz linear ultrasound transducer; Vivid S6, GE Healthcare ultrasound machine, Little Chalfont, UK). Ultrasound scanning was performed with the probe in paramedian sagittal plane. First, the ribs and the intercostal spaces were identified $4-5 \mathrm{~cm}$ lateral to the spinous process of the corresponding vertebral bodies (Figure 1A). In this view, the round contours of the ribs were visualized with the pleural line between them located $\sim 0.5 \mathrm{~cm}$ deeper. The scanning probe slid from lateral to medial (maintaining sagittal orientation) until the transverse processes (TPs) then came into view. The transition was seen as a "step down" change. In contrast with ribs, the appearance of TPs was more rectangular with a pleural line located deeper (Figure 1B). In this view, the PV space was visualized with the costotransverse ligament (CTL) located above it. Advancing the probe more medially, the transition of TPs to vertebral laminae was then visualized (Figure 1C), followed by the view of the adjacent vertebral laminae (Figure 1D). The laminae, targeted with the retrolaminar approach, appeared sonographically as flat continuous interrupted "notched" hyperechoic structures, constituting the location of optimal needle-bone contact end point.

An in-plane approach using an 18 gauge Tuohy needle (Braun Medical Inc, Bethlehem, PA, USA) was employed. The needle was introduced at the cephalic end of the ultrasound probe aiming caudally and was advanced until contact with vertebral lamina was achieved and confirmed both by imaging and "by feel" (Figure 1D). Then, the local anesthetic/dye mixture was injected through the needle under real-time ultrasound visualization. The criterion for assessment of correct spread of the injectate was creating a plane/hypoechoic space between the lamina and the deep paraspinous muscles.

Two hours after the procedure, the cadavers were dissected by first removing the anterior thoracic wall and then carefully 

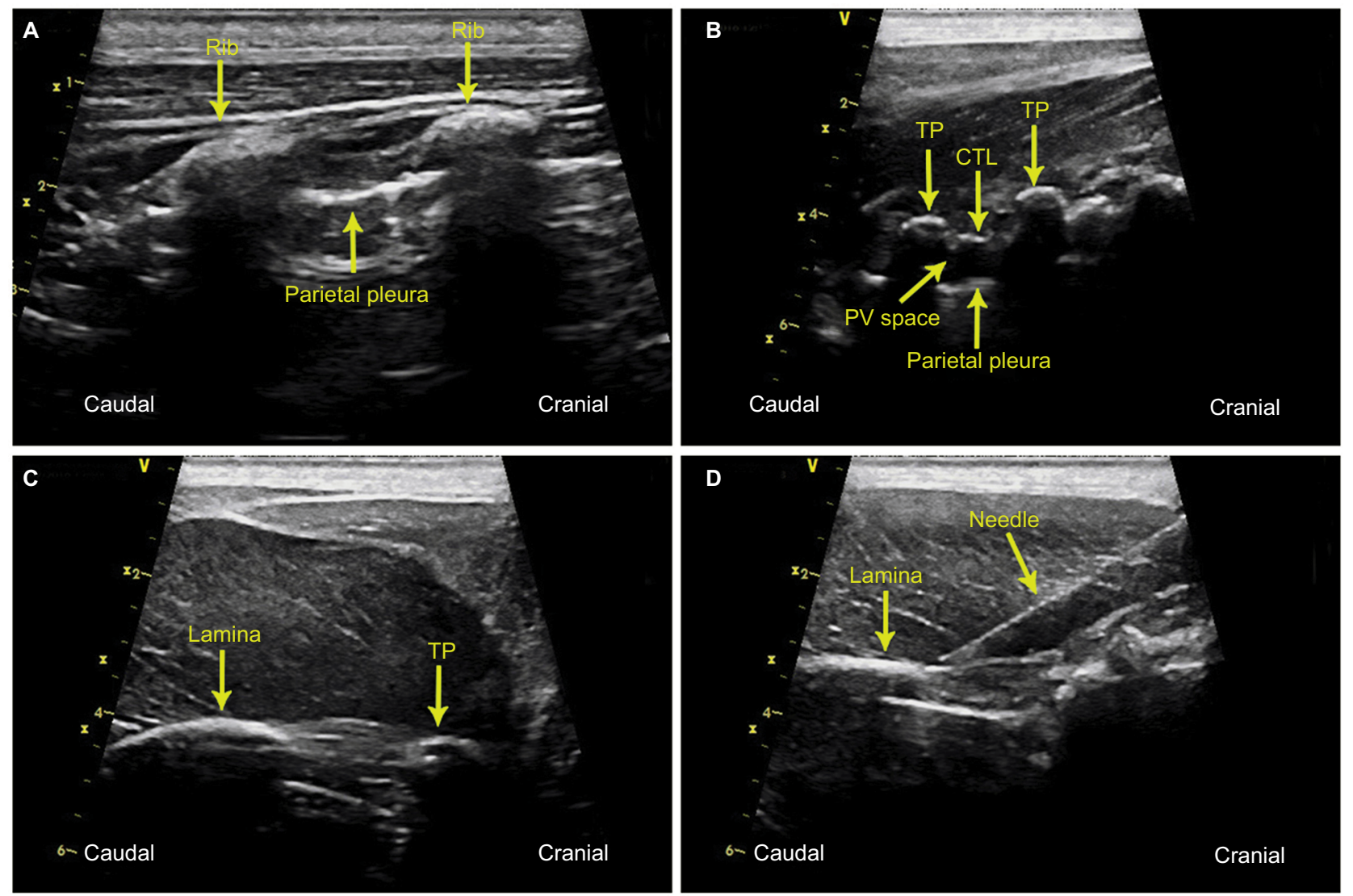

Figure I Sagittal paraspinal sonography views in fresh porcine cadavers, obtained by paramedian sagittal plane ultrasonography in left recumbent position with a 9 L-RS linear transducer, ultrasound machine (Vivid S6, GE Healthcare).

Notes: (A) Ultrasonography view of the ribs; (B) Ultrasonography view of the TPs; (C) Ultrasonography view of the transition from TPs to vertebral lamina; (D) Ultrasonography view of the vertebral lamina and the block needle coming into contact with the lamina prior to injection.

Abbreviations: TP, transverse process; CTL, costotransverse ligament; PV, paravertebral.

eviscerating the thorax to allow an unobstructed view of the posterior thoracic wall, including PV and intercostal spaces. The pleura was carefully inspected for evidence of needle penetration or intrapleural contrast. Afterwards, the dissected animals were deep-frozen to a temperature $-30^{\circ} \mathrm{C}$ and crosssection cuts (around $1 \mathrm{~cm}$ thick) of the posterior thoracic wall ( $16 \mathrm{~cm}$ above and below the injection point) were performed to evaluate the injected spread.

An independent observer, blinded to the group allocation, evaluated the cross-sections. The criterion for PV spread of the injectate was identification of any dye anterior to the TPs and the vertebral laminae (PV space to parietal pleura). The spread of injectate within the PV space was measured in the cranio-caudal direction. The cross-sections were also inspected for epidural and contralateral PV spread.

Continuous variables were expressed as median (range) and categorical variables as $n(\%)$. This pilot study was not intended to test specific hypotheses and was not powered for such tests; therefore, our findings are presented with descriptive statistics only.

\section{Results}

The retrolaminar space/tissue plane was easily identified in all porcine cadavers, and ultrasound images were comparable with human sonoanatomy (Figure 2 - healthy volunteer, sitting position, X-Porte ultrasound machine, HFL50XP 15-6 $\mathrm{MHz}$ linear transducer, SonoSite, Bothell, WA, USA). All injections fulfilled the criteria for successful placement, as real-time visualization during injection demonstrated the spread of the injectate between the lamina and the deep paraspinous muscles in all cadavers. The median distance from skin to the laminae (at the site of needle-lamina contact) was $3 \mathrm{~cm}$ (range, 2.5-3.5 cm). In comparison, the corresponding PV spaces were identified at depth of $5 \mathrm{~cm}$ (range, 4-5 cm).

In the high-volume group (Figure 3A, red arrows), 2\% lidocaine/methylene blue spread from retrolaminar to PV space was observed in all animals ( 5 out of $5 ; 100 \%$ ). In the low-volume group (Figure 3B), no 2\% lidocaine/methylene blue spread to the PV space was detected (0 out of 5; $0 \%$ ). The median injectate spread in the PV space in cranio-caudal direction (high-volume group) was $7 \mathrm{~cm}$ (range, 6-16 cm). 

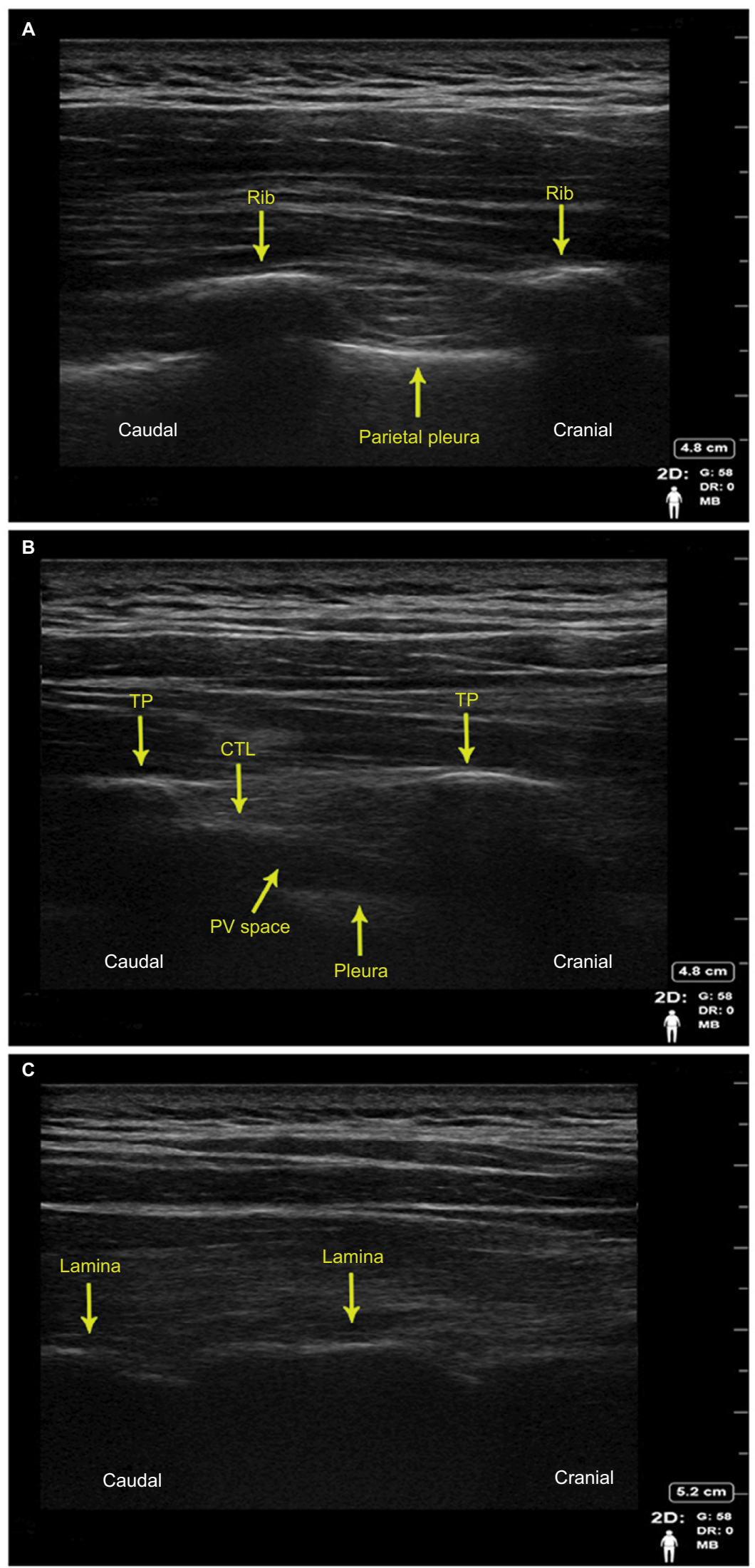

Figure 2 Human sonoanatomy images obtained by paramedian sagittal plane ultrasonography in a healthy volunteer, sitting position, X-Porte ultrasound machine, HFL50XP 15-6 MHz linear transducer (SonoSite).

Notes: (A) Proximal intercostal view; (B) "Classical” PV; and (C) Retrolaminar view.

Abbreviations: TP, transverse process; CTL, costotransverse ligament; PV, paravertebral. 
A

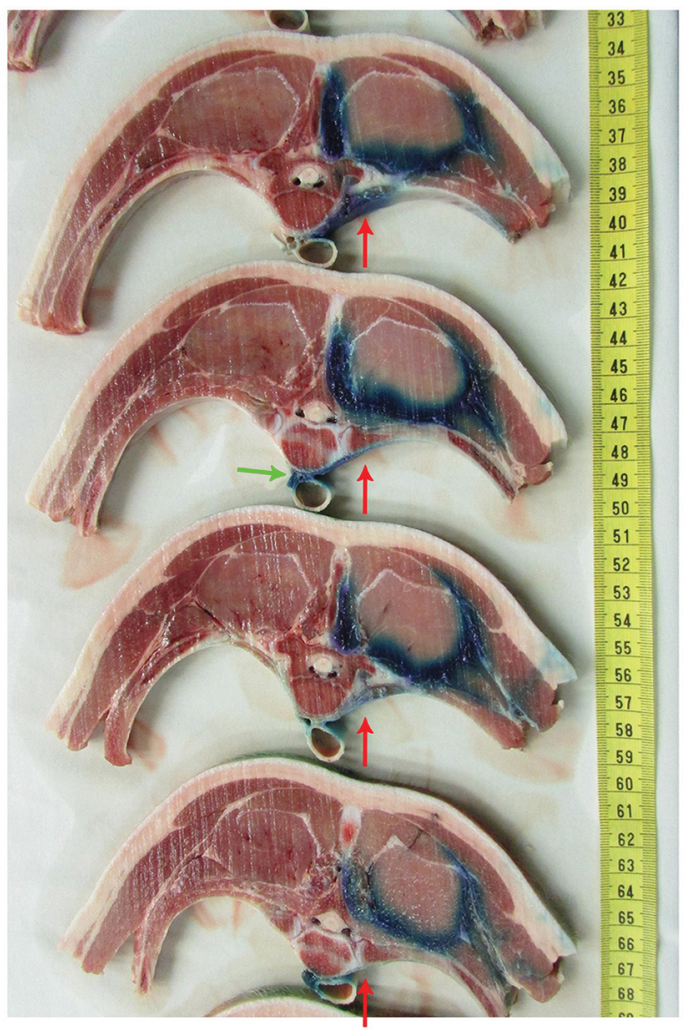

B

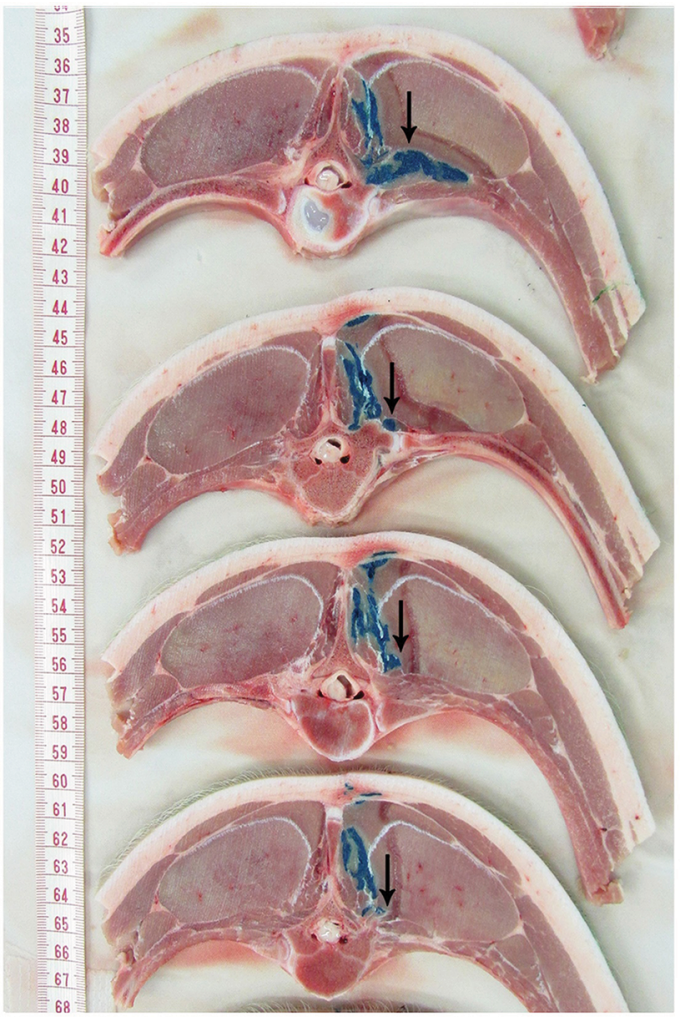

Figure 3 Cross-section cuts after porcine cadaveric dissection and removal of the intrathoracic viscera.

Notes: (A) High-volume group, red arrows - the injectate spreads from the retrolaminar to the PV space; green arrow - the injectate spreads anterior to the vertebral body. (B) Low-volume group - the injectate was observed between the vertebral laminae and the paraspinal muscles (black arrows). No spread into the paravertebral space was observed.

Abbreviation: PV, paravertebral.

There was no epidural or contralateral PV spread of the injectate in any of the studied specimens. In two specimens of the high-volume group, there was spread of the injectate anterior to the vertebral body, crossing its midline (Figure 3A, green arrow). There was no evidence of parietal pleural punctures or pleural injectate spread in any of the cadavers.

\section{Discussion}

This observational anatomical study on pigs demonstrates that the injectate distribution from the retrolaminar plane to the PV space is volume dependent, suggesting that high volumes would be required to achieve effective regional anesthesia and analgesia that would be consistent with PVB. This is in agreement with the previously published clinical reports and case series where relatively high volumes of local anesthetics were needed to accomplish a successful block using the retrolaminar approach. ${ }^{12-16}$

We postulate that higher volumes of local anesthetics, introduced quickly into the relatively noncompliant retrolaminar space/tissue plane, generate pressures, which might allow for trickling of the injectate into the PV space through the anatomical communications between the two spaces. This hypothesis reflects the evolution of the classical anatomical concept of the PV space described as a closed triangular space situated between the head and neck of the ribs which has three walls: the posterior wall formed by the superior CTL, the anterolateral wall consisting of the parietal pleura, and the medial wall of the lateral surface of the vertebral body and disk. Jüttner and Pfeiffer have claimed that the CTL is a porous structure and as such cannot be an obstacle for local anesthetic penetration into the PV space. ${ }^{10,11}$ Since the CTL structure is no different from other ligaments, it is unlikely to be porous. However, the dorsal ramus of the spinal nerve exits posteriorly to innervate the paraspinal muscles through a medial aperture of the superior CTL. This anatomical connection between the retrolaminar and PV spaces can be a pathway for injectate seepage. Another possibility can be that the fluid tracks anteriorly through the looser tissues just lateral to the facet joints, ${ }^{13}$ especially in the case of higher injection or compartment pressure. As many 
ultrasound-guided retrolaminar block case reports have been in trauma patients, ${ }^{13-15}$ one logical hypothesis would be that the disruption of ribs, ligaments, and fascia plays an important role in facilitating the access of the local anesthetic to the true PV space. In our study, however, the thoracic cage was intact, which points to a real anatomical communication between retrolaminar and PV spaces rather than a traumatic disruption. In this context, the clinical indications expanded beyond trauma, and so the reported success of this block in treating pain after mastectomy, thoracic, and abdominal surgery can be better explained. Moreover, the observed craniocaudal spread within the PV space suggests a potential for multisegmental nerve coverage based on measured distances. However, we recognize the limitations of our study model and design - volume-to-block extent relationship should be investigated further both anatomically and clinically.

In our study, there was no evident epidural or contralateral PV spread of the injectate. However, we observed spread of the injectate anterior to the vertebral body, reaching its contralateral half in two specimens of the high-volume group. Spread across the midline of the vertebral body of the contrast in vivo ${ }^{18}$ or dye in cadavers ${ }^{19,20}$ has already been reported in the literature. An anatomical basis for this observation was provided by Karmakar et al, ${ }^{18}$ who postulated that the extrapleural compartment of the thoracic PV space (between the parietal pleura and the anterolateral, extrapleural compartment of the endothoracic fascia ${ }^{21}$ ) along the subserous fascial plane acts as a channel of communication for the PV spaces on either side anterior to the vertebral bodies.

The other beneficial information obtained from our study is that the ultrasound anatomy of the porcine retrolaminar and PV space is very similar to that of a human, which is important for further experimental studies as availability of fresh frozen human cadaver material is limited. Close anatomical similarity between the human and porcine spine has already been demonstrated in other studies, which have neither been focused on PV and retrolaminar space nor on the evaluation of ultrasound comparability. ${ }^{17,22-24}$ In comparison, Figure 2 demonstrates the human sonoanatomy of the proximal intercostal, "classical" PV and retrolaminar views, respectively, obtained in a sagittal plane. The apparent morphological similarities do not exclude the possibility that significant differences might exist in tissue pliability and pathways for fluid spread between compartments and tissue planes.

Our study has important limitations. Although fresh porcine cadavers were used and the time from euthanasia to completion of dissection was limited to $3 \mathrm{~h}$, tissue changes associated with death are inevitable. ${ }^{25}$ In that context, the spread of injectate can be theoretically facilitated or impaired, in comparison with conditions in vivo. In this respect, the specimens in both groups (low- and high-volume) were subjected to the same conditions. Second, it could be expected that relatively fast manual injections of high-volume boluses may result in both high injection pressures and also high tissue "compartment" pressures in the relatively noncompliant retrolaminar space/tissue plane. However, injection pressures and injection rates were neither measured nor compared between the two study groups. Third, from a statistical point, our study has all drawbacks of descriptive statistics in comparison with inferential statistics. However, there is an obvious difference in spread to the PV space ( $0 \%$ vs $100 \%)$ from which we may draw our conclusions. Last but not least, direct translation of our results to humans maybe limited by the anatomical differences between the species, but basic principles might still be relevant.

Finally, we must submit that it is unclear if the anatomical spread of local anesthetic into the PV space should be viewed as the only surrogate for clinically successful thoracic anesthesia and analgesia. On one hand, Luyet et $\mathrm{al}^{26}$ reported a significant discrepancy between the position of intended PV catheters and clinical analgesia. On the other hand, the newly reported case series of erector spinae plane block producing extensive thoracic analgesia ${ }^{27}$ further challenges conventional anatomical explanations.

\section{Conclusion}

In summary, under the conditions of our study, the spread of injectate from retrolaminar to PV space in porcine cadaver model is volume dependent. In clinical terms, this supports the notion of improved efficacy of the ultrasound-guided retrolaminar block when high volumes of local anesthetics are used.

\section{Acknowledgments}

For their assistance with the study, we thank Ivan Blazinovic, Stane Kristl, Majda Crnak-Maasarani, Marko Slak, Natasa Pollak, Friderik Stendler, Milan Stevanec, and Andreja Vidmar from the Institute of Anatomy, Faculty of Medicine; University of Ljubljana, Ljubljana, Slovenia. This work was funded by the Slovenian Research Agency (P3-0043-0381) and the tertiary funding of the Clinical Department of Anesthesia and Intensive Therapy, University Medical Center Ljubljana, Slovenia. Results of the study were presented as abstract at the 34th Annual ESRA Congress, Ljubljana, Slovenia, September 2-5, 2015. 


\section{Disclosure}

The authors report no conflicts of interest in this work.

\section{References}

1. Bulger EM, Arneson MA, Mock CN, et al. Rib fractures in the elderly. J Trauma. 2000;48:1040-1046.

2. Mohta M, Verma P, Saxena AK, et al. Prospective, randomized comparison of continuous thoracic epidural and thoracic paravertebral infusion in patients with unilateral multiple fractured ribs: a pilot study. J Trauma. 2009;66:1096-1101.

3. Ho AM, Karmakar MK, Critchley LAH. Acute pain management of patients with multiple fractured ribs: a focus on regional techniques. Curr Opin Crit Care. 2011;17:323-327.

4. Pintaric TS, Potocnik I, Hadzic A, et al. Comparison of continuous thoracic epidural with paravertebral block on perioperative analgesia and hemodynamic stability in patients having open lung surgery. Reg Anesth Pain Med. 2011;36:256-260.

5. Lucas SD, Higdon T, Boezaart AP. Unintended epidural placement of a thoracic paravertebral catheter in a patient with severe chest trauma. Pain Med. 2011;12:1284-1289.

6. Richardson J, Lonnqvist PA, Naja Z. Bilateral thoracic paravertebral block: potential and practice. Br J Anaesth. 2011;106:164-171.

7. Cowie $\mathrm{B}, \mathrm{McGlade} \mathrm{D}$, Ivanusic J, et al. Ultrasound guided thoracic paravertebral blockade: a cadaveric study. Anesth Analg. 2010;110:1735-1739.

8. Abrahams M, Derby R, Horn JL. Update on ultrasound for truncal blocks: a review of the evidence. Reg Anesth Pain Med. 2016;41:275-288.

9. Luyet C, Herrmann G, Ross S, et al. Ultrasound-guided thoracic paravertebral puncture and placement of catheters in human cadavers: where do catheters go? Br J Anaesth. 2011;106:246-254.

10. Pfeiffer G, Oppitz N, Schone S, et al. Analgesia of the axilla using a paravertebral catheter in the lamina technique. Anaesthesist. 2006;55:423-427.

11. Juttner $T$, Werdehausen $R$, Hermanns $H$, et al. The paravertebral lamina technique: a new regional anesthesia approach for breast surgery. J Clin Anesth. 2011;23:443-450.

12. Zeballos JL, Voscopoulos C, Kapottos M, et al. Ultrasound-guided retrolaminar paravertebral block. Anaesthesia. 2013;68:649-651.

13. Voscopoulos C, Palaniappan D, Zeballos J, et al. The ultrasound-guided retrolaminar block. Can J Anesth. 2013;60:888-895.
14. Yoshida H, Yaguchi S, Matsumoto A, et al. A modified paravertebral block to reduce risk of mortality in a patient with multiple rib fractures. Am J Emerg Med. 2015;33:735.

15. Yoshida H, Yaguchi S, Chiba N, et al. Ultrasound-guided lumbar retrolaminar block for acute lumbar trauma. Anaesth Intensive Care. 2015;43:528-529.

16. Murouchi T, Yamakage M. Retrolaminar block: analgesic efficacy and safety evaluation. J Anesth. 2016;30(6):1003-1007.

17. Damjanovska M, Cvetko E, Hadzic A, et al. Neurotoxicity of perineural vs intraneural extrafascicular injection of liposome bupivacaine in the porcine model of sciatic nerve block. Anaesthesia. 2015;70: $1418-1426$.

18. Karmakar MK, Kwok WH, Kew J. Thoracic paravertebral block: radiological evidence of contralateral spread anterior to the vertebral bodies. Br J Anaesth. 2000;84:263-265.

19. Lönnqvist PA, Hildingsson U. The caudal boundary of the thoracic paravertebral space. A study in human cadavers. Anaesthesia. 1992;47: 1051-1052.

20. Tenicela R, Pollan SB. Paravertebral-peridural block technique: a unilateral thoracic block. Clin J Pain. 1990;6:227-234.

21. Stopar PT, Veranic P, Hadzic A, et al. Electron-microscopic imaging of endothoracic fascia in the thoracic paravertebral space in rats. Reg Anesth Pain Med. 2012;37:215-218.

22. Busscher I, Ploegmakers JJ, Verkerke GJ, et al. Comparative anatomical dimensions of the complete human and porcine spine. Eur Spine J. 2010;19:1104-1114.

23. Sheng SR, Wang XY, Xu HZ, et al. Anatomy of large animal spines and its comparison to the human spine: a systematic review. Eur Spine J. 2010;19:46-56.

24. Bozkus H, Crawford NR, Chamberlain RH, et al. Comparative anatomy of the porcine and human thoracic spines with reference to thoracoscopic surgical techniques. Surg Endosc. 2005;19:1652-1665.

25. Johnson RA, Lopez MJ, Hendrickson DA, et al. Cephalad distribution of three differing volumes of new methylene blue injected into the epidural space in adult goats. Vet Surg. 1996;25:448-451.

26. Luyet C, Siegenthaler A, Szucs-Farkas Z, et al. The location of paravertebral catheters placed using the landmark technique. Anaesthesia. 2012;67:1321-1326.

27. Forero M, Adhikary SD, Lopez H, et al. The erector spinae plane block: a novel analgesic technique in thoracic neuropathic pain. Reg Anesth Pain Med. 2016;4:621-627.
Journal of Pain Research

\section{Publish your work in this journal}

The Journal of Pain Research is an international, peer reviewed, open access, online journal that welcomes laboratory and clinical findings in the fields of pain research and the prevention and management of pain. Original research, reviews, symposium reports, hypothesis formation and commentaries are all considered for publication.

\section{Dovepress}

The manuscript management system is completely online and includes a very quick and fair peer-review system, which is all easy to use. Visit http://www.dovepress.com/testimonials.php to read real quotes from published authors. 